\title{
An Adaptive Technique To Maximize Lossless Image Data Compression of Satellite Images.
}

\author{
Robert J. Stewart ; Y. M. Fleming Lure * C. S. Joe Liou
}

\begin{abstract}
Data compression will play an increasingly important role in the storage and transmission of image data within the NASA science programs as the Earth Observing System comes into operation. It is important that the science data be preserved at the fidelity the instrument and satellite communication systems were designed to produce. Lossless compression must therefore be applied, at least, to archive the processed instrument data. In this paper we present an analysis of the performance of lossless compression techniques and develop an adaptive approach which applied image remapping, feature-based image segmentation to determine regions of similar entropy, and high-order arithmetic coding to obtain significant improvements over the use of conventional compression techniques alone. Image remapping is used to transform the original image into a lower entropy state. Several techniques were tested on satellite images including differential pulse code modulation, bi-linear interpolation, and block-based linear predictive coding. The results of these experiments are discussed and trade-offs between computation requirements and entropy reductions are used to identify the optimum approach for a variety of satellite images. Further entropy reduction can be achieved by segmenting the image based on local entropy properties then applying a coding technique which maximizes compression for the region. Experimental results are presented showing the effect of different coding techniques for regions of different entropy. A rule-base is developed through which the technique giving the best compression is selected. The paper concludes that maximum compression can be achieved cost effectively and at acceptable performance rates with a combination of techniques which are selected based on image contextual information.
\end{abstract}

\footnotetext{
${ }^{1}$ The author is with Caelum Research Corp., Silver Spring, Md 20901, Phone: (301) 593-1748.
} 


\section{Introduction}

With a steadily growing use of imaging technology in almost all computerized scientific fields the need for better image compression for purposes of minimizing transmission time and storage space/cost is ever present. Lossless image compression which permits faithful reconstruction of the original image is important in maintaining accurate image archives of digitized documents, in remote senșing image storage and retrieval, and in medical imaging where loss of fidelity due lossy compression can compromise radiological diagnosis. The techniques for lossless compression basically attempt to re-code image data such that redundant elements are coded with the least number of bits possible by using the frequency of occurrence of elements to determine the number of bits to use to code the image [1] [2]. The amount of compression obtained is related to the degree of redundancy present in the image. Tu obtain higher compression over the basic approach, preprocessing techniques which attempt to decorrelate image pixel values [3] and source modeling techniques which attempt to use the context of local pixel values [4] have been tried. One- and two-dimensional discrete pulse code modulation (DPCM), [5] bi-linear interpolation [6], and hierarchical interpolation [7] techniques are typical of the decorrelation approaches that have been applied to improve image compression. Prior to coding an image, a statistical model of the image is needed which can be developed in a separate pass over the image or adaptively as the image pixels are being coded [8]. Zero-order models consider each pixel to be independent of its neighbors. Higher-order models collect statistics on sequences of adjacent pixels [9]. Higher-order statistical models provide better compression where images are smooth and regular and zero order models are more effective where the images have a large high frequency content [10]. Investigators have shown that combinations of these techniques when applied to particular classes of images provide improved compression, but no one combination is suitable for all images, particularly satellite images where the image texture can change dramatically over small spacial distances, from smooth desert regions to rough snow-capped mountains. Based on work done on lossless image compression for medical images by the authors [11] and others [12], it is believed that satellite imagery would best be compressed with multiple compression techniques which are adaptively selected based on properties of local image regions. Image regions which contain a large degree of prominent fine texture will not compress well because little data redundancy and inter-pixel correlation will exist. For such regions, the simplest least computationally expensive compression technique should be applied. For regions with smooth textures or regular patterns, image decorrelation and high-order modeling will produce the highest compression. For regions with characteristics between these two extremes, the most compression will be achieved with some combination of decorrelation and statistical modeling with the order of the statistics selected based on some measure of inter-pixel pattern repetition. The problem then becomes the identification of image features which can be used to select the decorrelation, modeling, and encoding techniques to give maximum compression at minimum "cost": Cost being a measure of the computational requirements for a given approach against the degree of improvement in compression achieved by using it. If such a feature set can be found, then the image to be compressed could be divided into arbitrarily small regions, the features calculated for each region, the regions classified according to the best compression techniques to apply, and then similarly classified regions compressed appropriately (Figure 1). A pipelined process of image analysis, feature ex- 
traction, classification, decorrelation followed by modeling and coding is shown in Figure 1. Parallelization is possible due to the region processing approach used. Feature extraction and region classification are performed in parallel on each image region. The classifier determines the correct compression approaches to used based on a decision tree approach using the calculated features. Image regions that will use a decorrelation preprocessor will be passed through that path, other regions will be processed by the modeler and coder appropriate to the type of region. The modeler and coder are serialized processes in this architecture because adaptive modeling will be used, that is, the statistical model is built as the image pixels are being compressed. All image regions that are similarly classified will be processed through the same modeler/coder path so that a unique statistical model is generated for each type of region. The modeler and coder will process all pixels in a region then proceed to the next region, and so on. This procedure preserves any two-dimensional correlation of pixel values and should maximize compression. To be able to decompress the image file, it is necessary that the compressed file output by this architecture contains a header which records a classifier identifier for each region. The image can be reconstructed by reading the region classifier identifier and decompressing the region through the reverse of the compression process.

The application of a decorrelation step prior to modeling and coding was also considered after proving the suitability of this approach without it. Decorrelating the image reduces pixel value variance [10] and therefore improves compression. Two decorrelation methods were used, DPCM and bi-linear interpolation. Both are relatively simple to implement with DPCM using one or more previous pixel values to predict the current pixel value, and the lattice points in a 2-dimensional kernel of pixels being used to predict the other kernel pixel values. In the case of bi-linear interpolation, the output is two data streams, a set of prediction errors, and the value of the lattice points for each non-overlapping 2-dimensional kernel in the image. The computational cost of the bi-linear interpolation process is considerably more than the DPCM approach, but the improvement in compression can be considerable for certain types of images.

\section{Image Analysis and Feature Extraction}

The key to the success of this adaptive scheme for compression lies in the selection of features which can usefully classify image regions into the optimum compression approach and thereby minimize the compressed file size. Their exist no theoretical foundation for determining the feature set that would provide optimum compression selection. An empirical approach was take to determine the best feature set: Satellite images were selected for compression which contained a large variety of textures. The images were divided into symmetrical regions and each region was compressed using several decorrelation and lossless compression techniques. Several features were calculated for each region. Using cluster analysis techniques to identify unique reions in feature space, a binary decision tree classifier was developed using features from a large variety regions. The features selected for the initial training of the classifier were determined by evaluation of the compression process and from previous work on medical image compression [11], [13]. There are several well known techniques for estimating the amount and degree of texture in an image, including co-occurrence matrices [14], sum and 


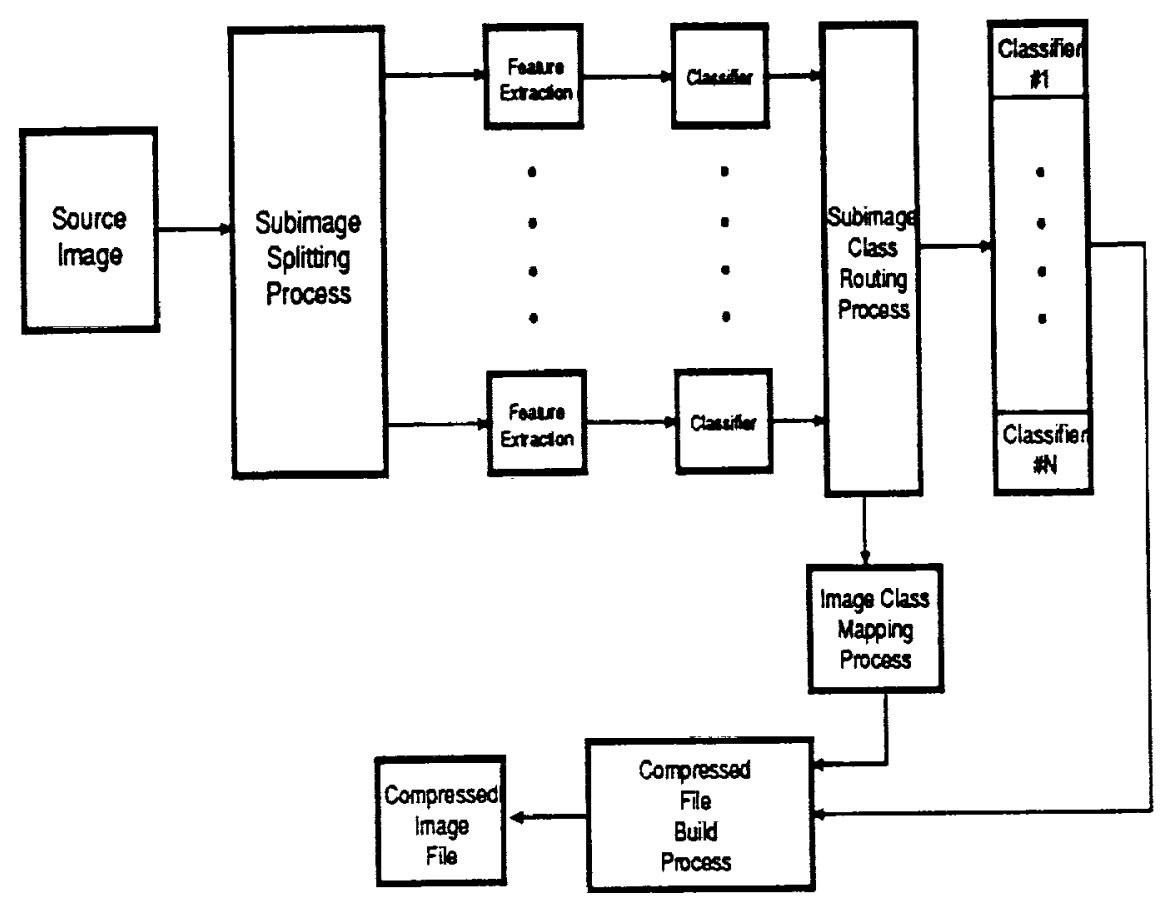

Figure 1: Architecture of the image compression approach.

difference matrices [15], and local grey-scale dependance matrices [16]. Due to the memory requirements of these approaches (at least the same amount as the original image), we elected to use an feature set less resource demanding. The compressibility of an image is related to the range and distribution of intensities in the image, the individual pixel entropy, and the degree of variability in local pixel intensity values. Based on this knowledge, the initial feature included:

Average pixel intensity - The mean over the region of the pixel values, selected so that regions similar brightness would be group together:

$$
\bar{x}=\frac{\sum_{i, j} p(i, j)}{N}
$$

where, $\mathrm{i}, \mathrm{j}$ are the rows and columns, respectively, in the $\mathrm{n} \times \mathrm{n}$ subimage, $\mathrm{N}=\mathrm{n}+\mathrm{n}$, and $p(i, j)$ the intensity of pixel $i, j$.

Pixel Intensity Variance - The variability in brightness over the region:

$$
P_{v}=\frac{\sum_{i, j}(p(i, j)-\bar{x})^{2}}{N}
$$

First-Order Entropy - The amount of pixel value redundancy present in the region:

$$
H=\sum_{i, j}\left(-P_{i, j} \log _{2} P_{i, j}\right)
$$


Average Run-length - The average value of the run-length sections generated by thresholding the subimage with the mean gray-level:

$$
R_{a}=\frac{\sum_{l, j} R_{l, j}}{M}
$$

where, $1, j$ are the rows and columns in an $m \times n$ subimage runlength matrix, $M=m+n$, and $R$ is the distance of the run length.

Run-length Variance - The variance in run lengths obtained by thresholding the subimage with the mean gray-level:

$$
R_{v}=\frac{\sum_{l, j}\left(R_{l, j}-R_{a}\right)^{2}}{M}
$$

The images analyzed in this process were all of sea-ice taken from spectral band 3 from

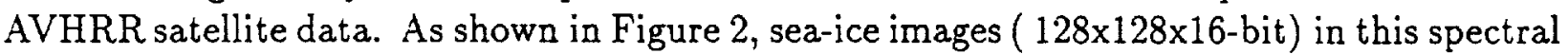
band provide a wide variety of textures from smooth to very rough. The compressibility of regions within this large image varies considerably: with the left, middle and right images in the Figure compressing to $35 \%, 39 \%$, and $28 \%$, respectively, when arithmetic coding is used with first-order modeling. As would be expected, the less textured images compress better.

\section{Decision Tree Classifier}

An unsupervised classifier method was used to form clusters in feature space, and cluster analysis [17] used to allocate class regions based on minimizing intra-class second-order moments. In the training process, subimages feature vectors containing the above features were calculated for a training set. As each subimage was processed, its feature vector was calculated, it's nearest neighbor in feature space located, the new centroid of the region calculated, and the moments of the vector in that class calculated. Class region boundaries were recalculated when the second moments for the region started to diverge. A binary decision tree was selected for classification using the Kolmogorov-Smirnov test [18] to determine the threshold value for each feature at each node in the decision tree. This results in the selection of a feature at each node which has maximum separation from the nearest neighbor in feature space. Figure 4 shows a representation of a binary decision tree based on the features identified in the previous section.

The classifier output is a class identifier for the subimage processed. This is then used to create a map of the subimages within the image. The map is used as header information, prepended to the compressed image file, and used by a decompression process to recover the original image. 


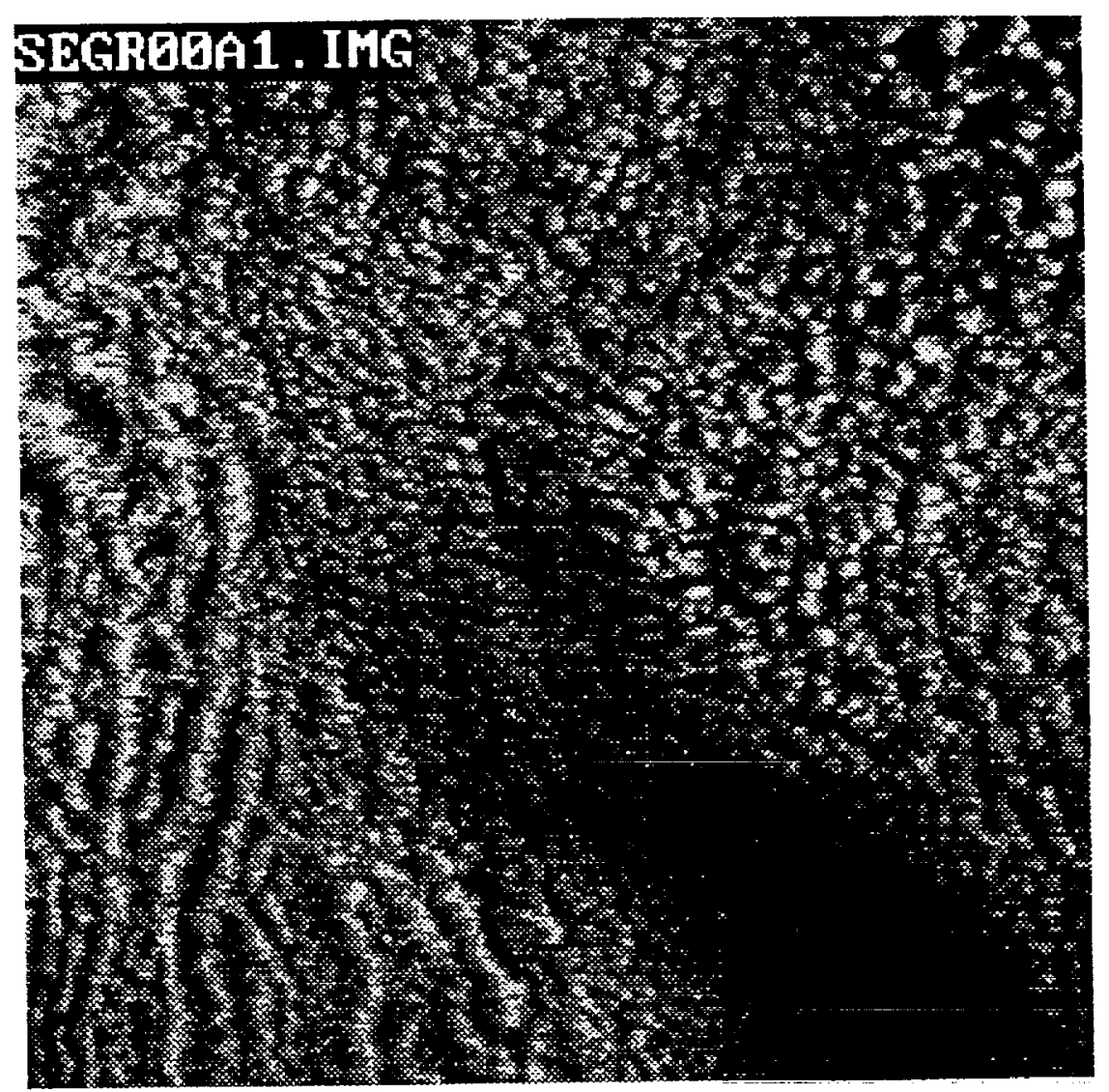

Figure 2: A $512 \times 512 \times 16$-bit sea-ice image showing typical texture variety 

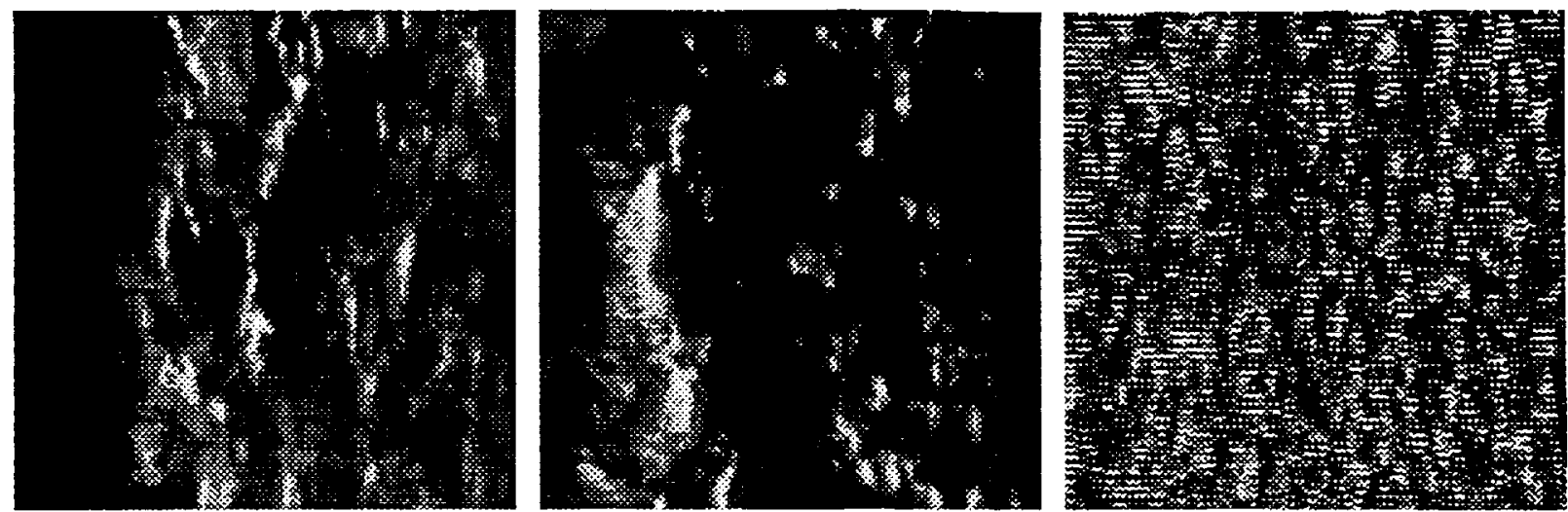

Figure 3: Sea-ice images showing light and dark evenly textured regions, and a region with high edge content

\section{Compression Results}

We selected several compression methods based on their ability to compress medical images. These were : Lempel-Ziv dictionary coding with a 15-bit code [19] (LZ), Huffman coding with adaptive modeling $(\mathrm{HC})$ arithmetic coding with static modeling ( $\mathrm{Ar}$ ) arithmetic coding with zero-order modeling (Ar-0) arithmetic coding with adaptive first-order modeling (Ar-1) arithmetic coding with adaptive second-order modeling (Ar-2)

We ran sixteen $512 \times 512 \times 16$-bit sea ice images through each of these processes to obtain a baseline of performance for each compression method. Table 1 shows the results of this baseline for three types of texture: Smooth regions with very little or gradual changes in textures; moderately textured regions similar to the middle image in Figure 3; and, regions including a high density of gray-scale variations. Next images were divided into $64 \times 64 \times 16$ bit subimages and the compression methods run on each subimage. The compression results were examined to determine the best compression method for each of the subimages. The best compression technique was always one from LZ, Ar- 0, Ar-1, or Ar-2 with a bilinear interpolation decorrelation preprocessing step. The number of classes $(k)$ to be considered was then taken as four. The feature set mentioned above was extracted for each image and cluster analysis performed for the subimages from the entire image set. An automatic classifier was built using the previously mentioned procedure and a binary decision tree was generated. The $16512 \times 512$ inages were then run through the adaptive process shown in Figure 1 and the compression values shown in Table 2 obtained.

\section{Conclusions}

The adaptive application of a variety of compression techniques on satellite images as opposed to applying one technique appears to give an improvement in lossless compression in order of 15 we have presented to select the lossless compression technique is to calculate features of subimages with the image and use then feature values in a binary decision tree to select the best compression technique for that subimage. While these results by no means 


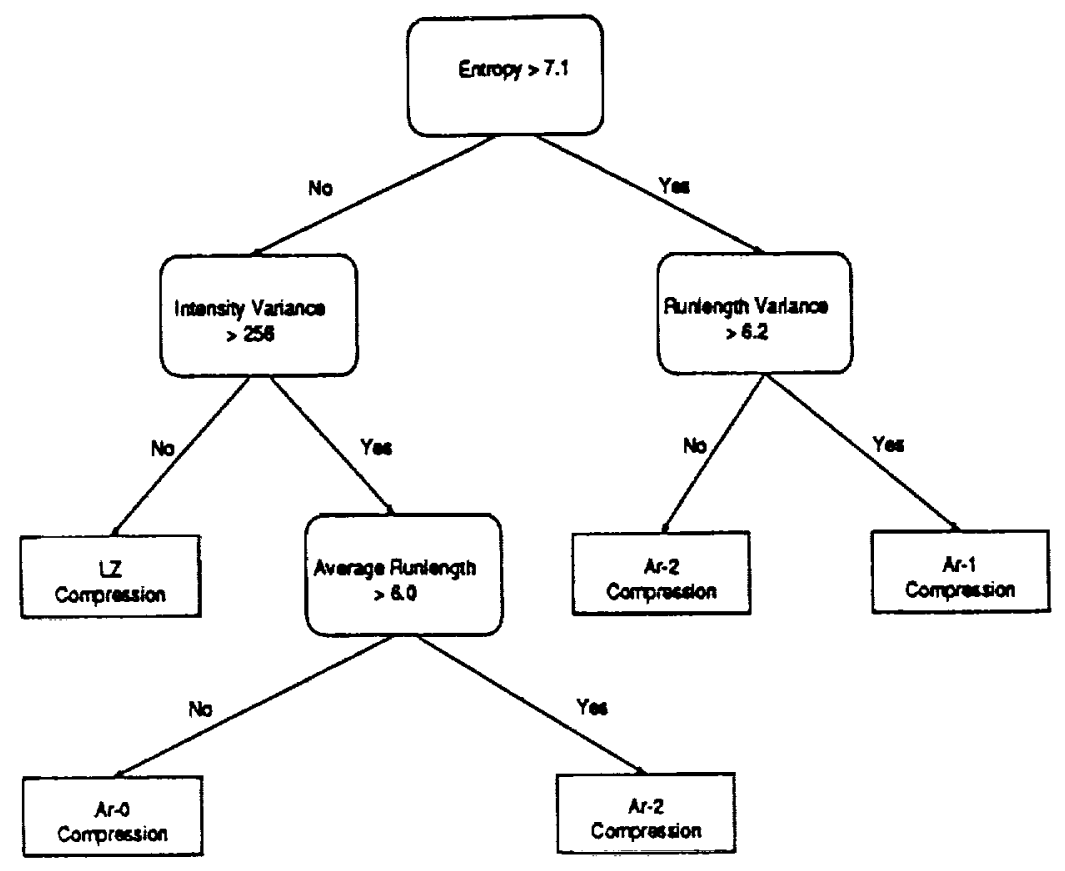

Figure 4: Binary decision tree to select the optimal compression method based on selected feature values

\begin{tabular}{|c|c|c|c|}
\hline $\begin{array}{c}\text { Compression } \\
\text { Method }\end{array}$ & $\begin{array}{c}\text { Smooth } \\
\text { Image }\end{array}$ & $\begin{array}{c}\text { Moderate } \\
\text { Image }\end{array}$ & $\begin{array}{c}\text { Granular } \\
\text { Image }\end{array}$ \\
\hline Huffman & 46 & 34 & 26 \\
\hline AR & 46 & 35 & 27 \\
\hline LZ & 54 & 46 & 35 \\
\hline Ar-0 & 51 & 47 & 44 \\
\hline AR-1 & 68 & 55 & 49 \\
\hline AR-2 & 68 & 51 & 43 \\
\hline
\end{tabular}

Table 1: The averaged values for the compression methods tested with compression expressed as a percentage of source/compressed file size

\begin{tabular}{|c|c|c|}
\hline $\begin{array}{c}\text { Smooth } \\
\text { Image }\end{array}$ & $\begin{array}{c}\text { Moderate } \\
\text { Image }\end{array}$ & $\begin{array}{c}\text { Granular } \\
\text { Image }\end{array}$ \\
\hline 71 & 62 & 56 \\
\hline
\end{tabular}

Table 2: Adaptive compression applied to the same images showing better compression than the best of Table 1 
imply that this approach will universally provide better compression for all satellite images, they do indicate that further work in expanding the test database is merited. Expansion of the compression approaches to include a decorrelation preprocessing step, as described previously should be undertaken. The feature set used to select the compression method was adequate, but attention needs to be paid to using the most cost/effective features from a processing point-of-view. It is believed that this compression approach using low-cost multiprocessing architectures and large secondary caches on each processor, will provide compression performance suitable of use in satellite imagery systems such as TRMM and EOS.

\section{References}

1 D. A. Huffman, " A Method for the construction of minimum-redundancy codes," Proc. IRE, vol. 40, pp. 1098-1101, Sept. 1952.

2 I. H. Witten, R. M. Neal, and J. G. Cleary, "Arithmetic coding for data compression," Commun. ACM, vol. 30, pp. 520-540, June 1987.

3 G. R. Kuduvalli and R. M. Rangayyan, "Performance analysis of reversible image compression techniques for high-resolution digital teleradiology," IEEE Trans. Med. Imaging, vol. 11, no. 3, September 1992.

4 T. V. Ramabadran and Keshi Chen, "The use of contextual information in the reversible compression of medical images," IEEE Trans. Med. Imaging, vol. 11, no. 2., June 1992.

5 . R. C. Gonzales, and P. Wintz, Digital Image Processing. Reading, MA.: AddisonWesley, 1977, pp. 276-281.

6 M. Rabbani and P. W. Jones, "Image compression techniques for medical diagnostic imaging systems," Jnl. of Digital Imaging, vol. 2, no. 2, pp 65-78, May 1991.

7 . P. Roos, M. A. Viergever, M. C. A. Van Duke, and J. H. Peters, "Reversible intraframe compression of medical images," IEEE Trans. Med. Imaging, vol. 7, pp. 328-336, Dec. 1988.

8 J. Rissanen and G. G. Langdon, Jr., "Universal modeling and coding," IEEE Trans. Inform. Theory, vol. IT-27, pp. 12-23, Jan. 1981.

9 J. G. Cleary and I. H. Witten, "Data compression using adaptive coding and partial string matching," IEEE Trans. Comm., COM-32, no. 4, pp. 396-402, Apr. 1984.

10 P. G. Howard and J. S. Vitter, "New methods for lossless image compression using arithmetic coding," Proceedings of the Data Compression Conference, Snowbird, Utah, pp. 3-12, 1991. 
11 Y. M. F. Lure, C. S. J. Liou, R. J. Stewart, and J. J. Metzner: "Reversible compression of medical images using decomposition and decorrelation methods", Proc. 26th IEEE Annual Asilomer Conf. on Sig. Syst. and Comput., Invited paper, Montery, CA, Nov. $1-3,(1993)$.

12 O. Baudin, A. Baskurt, F. Dupont, R. Prost, and R Goutte, "Structural analysis and coding of multimodal medical images," Proc. SPIE, Medical Imaging 1993, Image Capture, Formatting, and Display, Vol. 1897, pp. 298 - 304, 1993.

13 R. J. Stewart, Y. M. F. Lure, and C. S. J. Liou, "An approach to achieve maximum lossless compression of medical images", submitted to Data Compression Conference, IEEE Computer Society, Snowbird, Utah, Mar., 29-31 (1994).

14 R. M. Haralick, "Statistical and structural approaches to texture analysis," Proc. IEEE, vol 67, May, 1979, pp. 786-804.

15 M. Unser, "Sum and difference histograms for texture classification," IEEE Trans. on Pattern Analysis \& Machine Intelligence," Vol. PAMI-8, no. 1, Jan. 1986, pp. 118-125.

16 C. Sun and W. G. Wee, "Neighboring gray level dependance matrix for texture classification," Computer vison, graphics and image processing, vol. 23, 1983, pp. 341-352.

17 A. Jain and R. Dubes, Algorithms for Clustering Data, Englewood Cliffs, NJ, PrenticeHall, 1988.

18 J. R. B Cockett, and J. A. Herrera, "Decision tree reduction," Jnl. of the ACM, Vol 37, no. 4 , Oct, 1990.

19 J. Ziv, and A. Lempel: "Compression of individual sequences via variable-rate coding", IEEE Trans. Inform. Theory, May 1978, Vol. IT-24, no. 5, pp. 530-536. 